\title{
Evacuation Planning In Natural Disaster Using Multi Objective Optimization Approach: The Rohingya Refugee Crisis in Bangladesh
}

\author{
Md. Ridwanur Rahman', Samia Zakir Sarothi', Tanvir Mustafy ${ }^{1}$ \\ ${ }^{1}$ Department of Civil Engineering, Military Institute of Science and Technology \\ Mirpur Cantonment, Dhaka, Bangladesh \\ ridwanurrahman56@gmail.com, zakirsamia@ce.mist.ac.bd,mustafy@ualberta.ca
}

\section{Introduction}

The Rohingya refugee is an alarming humanitarian crisis in the current world community. Majority of these refugees are living in Kutupalong-Balukhali, Cox's Bazar under high congestion and annual disasters (i.e. landslide, cyclones, flood) have put their life in great risk. From this perspective, the study aims on proposing location-allocation based evacuation plan for the Rohingya camp using three different multi objective optimization techniques namely AMOSA [1], NSGA-II [2] and NMGA [2]. GIS is also integrated with the optimization techniques. We selected Camp 8E of the Kutupalong-Balukhali Expansion Site as our case study area.

\section{Methodology}

To solve the optimization problem two objective functions are used, function to minimize the travel time and the capacity overburden respectively [3,4]. No constrain are considered while solving the optimization problem. To compare the performance of algorithms, equal population size 100 and iteration number 300 are maintained for all the algorithms. Required spatial and non-spatial data are collected from UNOCHA services [5]. For safe place to evacuate refugees, nearby cyclone shelter designated by proper authority, and schools and prayer halls which meets the suitability criteria of relevant international standards [6] are selected. Capacity of the shelter are found from available data or calculated using crowd density standards [7]. Population of the camp studied is aggregated into small blocks. Shortest distance from each block to each shelter are calculated using origin-destination cost matrix tool in ArcGIS. Finally, all the attributes are compiled to create a database.

\section{Results}

In order to compare the performance of the three algorithms in solving the evacuation problem [2,3], different criteria such as solution quality, convergence speed, running time and repeatability are used. Result shows, AMOSA works best in optimizing both cost function, the average and worst fitness values for both time and capacity function is lowest in AMOSA followed by NMGA and NSGA-II. AMOSA, NSGA-II and NMGA returns 40, 32 and 25 solutions respectively in the final Pareto fronts. Higher number of solution in AMOSA is attributed by its archiving capacity. In terms of convergence speed and its smoothness, AMOSA and NSGA-II outperformed NMGA. To assess the repeatability, each algorithms is run 20 times. Repeatability is examined through variance of the average normalized fitness values for both objective function. In case of time function these variance are 0.04, 0.045 and 0.055 for AMOSA, NGMA and NSGA-II respectively. For capacity function the variation follows same. NSGA-II $(330.23 \mathrm{sec})$ has the minimum average execution time of 20 runs followed by AMOSA $(720.78 \mathrm{sec})$ and NMGA $(950.54 \mathrm{sec})$.In order to evaluate the statistical difference between tested algorithms, Kruskal-Wallis test is conducted. The result depicts, there is significant difference in terms of convergence speed and optimizing the cost functions among the algorithms.

\section{Discussion}

Presenting an effective evacuation plan for the Rohingya refugees during the event of natural disaster is the main concern of our research. Considering the solution quality and repeatability, AMOSA and NMGA can be recommended for evacuation planning purpose. But in need of quick solution NSGA-II can be suggested. The method reviewed herein provides a set of multiple optimum solutions. Decision makers can chose from these options for better planning of the 
evacuation proposal. With proper parameter tuning, this study can be further extended for the whole KutupalongBalukhali extension site.

\section{References}

[1] Bandyopadhyay, S., Saha, S., Maulik, U., \& Deb, K. (2008). A simulated annealing-based multiobjective optimization algorithm: AMOSA. IEEE transactions on evolutionary computation, 12(3), 269-283.

[2] Deb, K., Pratap, A., Agarwal, S., \& Meyarivan, T. A. M. T. (2002). A fast and elitist multiobjective genetic algorithm: NSGA-II. IEEE transactions on evolutionary computation, 6(2), 182-197.

[3] Mustafy, T., El-Rich, M., Mesfar, W., \& Moglo, K. (2014). Investigation of impact loading rate effects on the ligamentous cervical spinal load-partitioning using finite element model of functional spinal unit C2-C3. Journal of biomechanics, 47(12), 2891-2903.

[4] Mustafy, T., Londono, I., Moldovan, F., \& Villemure, I. (2019). High impact exercise improves bone microstructure and strength in growing rats. Scientific reports, 9(1), 1-14.

[5] Rohingya Refugee Crisis. Available from: https://data.humdata.org/dataset.

[6] The Sphere Project: Humanitarian charter and minimum standards disaster response :The Sphere Handbook; The Sphere Project: Rugby, UK. 2001, Mc Connan (ed) Geneva.

[7] Markus Friberg, M.H., Mass evacuation - human behavior and crowd dynamics - What do we know? LUND UNIVERSITY LIBRARIES. 